\title{
Structuring Safety Requirements in ISO 26262 using Contract Theory
}

\author{
Jonas Westman $^{1}$, Mattias Nyberg ${ }^{2}$, and Martin Törngren ${ }^{1}$ \\ 1 Royal Institute of Technology (KTH) \\ 2 Scania
}

\begin{abstract}
ISO 26262 - "Road vehicles-Functional Safety" is a standard for the automotive industry, administered in an attempt to prevent potential accidents due to systematic and random failures in the Electrical/Electronic-system. ISO 26262 is based on the principle of relying on safety requirements as the main source of information to enforce correctness of design. We show that the contract theory from the SPEEDS FP6 project provides a suitable foundation to structure safety requirements in ISO 26262. Contracts provide the necessary support to separate the responsibilities between a system and its environment by explicitly imposing requirements on the environment as assumptions, in order to guarantee the safety requirements. We show this by characterizing two levels of safety requirements with contracts for an industrial system where we also show how contract theory supports the verification of consistency and completeness of safety requirements.
\end{abstract}

\section{Introduction}

The standard ISO 26262-"Road vehicles-Functional Safety" [1] is, in essence, a domain-specific systems engineering approach with a focus on functional safety. ISO 26262 is based on the principle of relying on safety requirements as the main source of information to enforce correctness of design and implementation throughout the development process. A system and its elements are in ISO 26262 characterized by being logically and technically separated from their environment in the form of a detailed interface specification and separation of responsibilities. Although not mentioned explicitly, these principles are similar to the notion of a contract [2], namely: based on a well-defined system boundary, the responsibilities between an environment and a system are split into a guarantee that models desired properties of a system, under the influence of an assumption, modeling expected properties of an environment. In this paper, we explore a possibility to capitalize on this similarity, by using contracts to structure safety requirements in ISO 26262.

Out of three contributions, the first contribution is that we show that the theory of contracts can enrich safety specifications as it provides the necessary support to separate the responsibilities between a system and its environment by explicitly imposing requirements on the environment as assumptions, in order to guarantee the safety requirements. We show this by using the theory of contracts 
from the SPEEDS FP6 project $^{3}$ to characterize two levels of safety requirements for a real industrial system. Secondly, we show that the theory of contracts provides a foundation to argue for, and verify properties of safety requirements such as consistency and completeness as required by ISO 26262 . Thirdly, we show that a modification to the contract theory where assumptions can model properties of an environment that are not limited to the system boundary is needed, in order to conform with the principles in ISO 26262.

Several publications associated with the project CESAR ${ }^{4}$, see e.g. [3] and [4], discuss the use of contracts with respect to requirements engineering and safety standards, including ISO 26262, in general. In [5], this connection is elaborated on and a few examples (although not automotive) are shown where contracts could in fact be useful with respect to ISO 26262. However, none of [3], [4] nor [5] apply the theory on a real industrial system and mainly hypothesize about its usefulness when developing safety-critical systems. In this paper, we go in to further depths, showing an explicit use of contracts with respect to ISO 26262 by characterizing safety requirements as contracts for the Fuel Level Display (FLD)-system, present on all Scania vehicles.

The link between requirements engineering and contract theory is touched upon in [6-8], and more notably in [9], where properties of requirements, e.g. consistency, are described in a context of contracts. However, none of [6-9] address properties of safety requirements as described in ISO 26262 and the notion of completeness is not addressed to a full extent. In this paper, we establish a more elaborate connection between requirements engineering and contract theory by showing how consistency and completeness of safety requirements in ISO 26262 can be ensured through properties of contracts.

\section{Illustrative Example - The Fuel Level Display-system}

In this section, we introduce the illustrative example that will be used in Sec. 3 to exemplify a case where ISO 26262 relies on contract-inspired principles, and also in Sec. 5 to characterize safety requirements in ISO 26262 by contracts.

The FLD-system provides an estimate of the fuel volume in the fuel tank to the driver along with a warning if the fuel volume drops below a predefined value. The functionality provided by the FLD-system is distributed across three Electronic Control Unit (ECU)-systems, i.e. an ECU with sensors and actuators, in the Electronic/Electrical (E/E)-system: Engine Management System (EMS), Instrument Cluster (ICL), and Coordinator (COO). The ECU-systems also interact with the fuel tank that is outside of the E/E-system. COO estimates the fuel volume in the tank by relying on the output of a Kalman filter that, in turn, relies on a signal of a sensor measuring the fuel level in the tank and an estimate of the current fuel consumption provided by EMS, as inputs. The estimated fuel volume is sent over CAN to ICL, where it is displayed to the driver along with a warning if the fuel volume in the tank is below $10 \%$.

\footnotetext{
3 http://www.speeds.eu.com/

${ }^{4}$ http://www.cesarproject.eu/
} 
A development according to ISO 26262 revolves around an item which is in [1] described as "a system that implements a function at a vehicle level". For the analysis in this paper, COO, CAN, and ICL are chosen to be the item, as shown in Fig. 1, where we also illustrate the system boundary of the item.

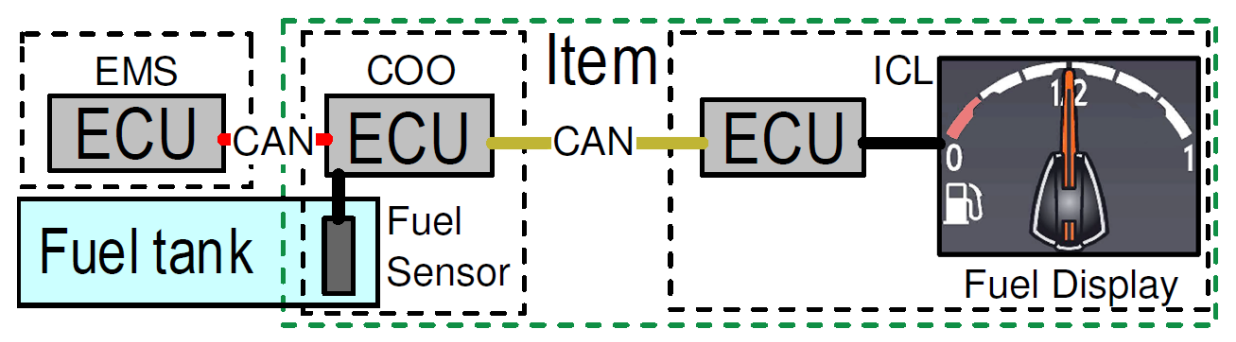

Fig. 1. System architecture of the FLD-system. The blocks represent actual ECUs, the fuel sensor, the fuel tank, and the display. The connectors represent physical cables. The borders (cross-hatched lines) represent the ECU-systems and the item.

\section{Motivation - Contract-Inspired Principles in ISO 26262}

We will as a motivation, in the following, present two rather explicit cases where ISO 26262 relies on principles similar to those of contracts from: 3-5 Item definition; and 10-8 Safety element out of context in [1].

Item Definition Prior to forming safety requirements, ISO 26262 first requires a description of the item as presented in 3-5 Item definition in [1]. We consider one of its requirements and apply it to the FLD-system, i.e. requirement 5.4.2:

"The boundary of the item, its interfaces, and the assumptions concerning its interaction with other items and elements, shall be defined considering: ... d) functionality required by other items, elements and the environment; e) functionality required from other items, elements and the environment..." [1]

Concerning sub-requirement 5.4.2d); a basic functionality of the FLD-system is to provide an accurate estimation of the fuel volume to the driver, who is part of the environment. We can formalize this functionality by the requirement: the indicated fuel volume, shown by the fuel gauge, shall not deviate more than \pm 5 percent from the actual fuel volume in the tank. However, in order for the item to be able to guarantee this functionality, the item needs information regarding the fuel consumption, which is provided by EMS, external to the item. Hence, concerning requirement 5.4.2e), we impose a requirement on EMS as an assumption: the estimated fuel consumption, provided by EMS, does not deviate more 
than \pm 1 percent from the actual fuel consumption. In conclusion, the item definition includes an interface specification with clear separation of responsibilities between the environment and the item - much like a contract.

System Element out of Context A System Element out of Context (SEooC) is a safety-related (i.e. assumed to be required to implement a safety requirement) element which is developed in isolation, that is, without the context of a specific item. Therefore, assumptions are made on the context of a SEooC, in the form of requirements that are likely to be allocated to its environment. The difference between a regular element (part of an item) and a SEooC is that a SEooC makes assumptions on a general environment while an element is to be integrated in a specific environment. The concept of SEooC addresses the need of subcontracting - an important aspect since companies in the automotive industry tend to rely on sub-systems developed external to the company. The concept of SEooC is similar to a description of contracts in a context of a distributed systems development environment where each supplier is given a design task in the form of a guarantee, subject to constraints under the responsibility of other actors of the company/supplier chain that are offered to this supplier as assumptions [2].

\section{The Contract Theory of SPEEDS}

The original use of contracts [10] as a pair of pre- and post-conditions as state predicates $[11,12]$ has been extended from software to e.g. Component-Based Design and hardware $[13,14]$. In this paper, we choose to apply the contract theory of SPEEDS. The reason for this is that ISO 26262 is centered on the development of E/E-Systems, which encompasses both hardware and software. In the contract theory of SPEEDS, contracts are formed for Heterogeneous Rich Components (HRCs) [15], which can represent entities of software, hardware, mechanical, etc. while the other approaches are typically used only in software.

In the following sections, we will hence present the theory of contracts as described in [2],[6], [9],[8] and [7] with inspiration from [16], [17], [18], and [19]. The intent is to present the theory in accordance with these papers; however, there might be slight deviations from the original papers since only a subset deemed relevant for the present paper is presented.

The presented theory of contracts will be used in Sec. 5.1 and 5.2 to model the architecture of the FLD-system and characterize safety requirements as contracts, and then in Sec. 5.4 to support the verification of requirements properties.

\subsection{Assertions and Runs}

Let $P=\left(x_{1}, \ldots, x_{N_{P}}\right)$ be an ordered set of variables where each variable is a function of time. Consider a trajectory of values assigned to a variable $x_{i}$ in $P$ over a whole time window. A tuple of such trajectories, one for each variable in $P$, is called a run for $P$. An assertion $B$ over $P$ is a set of runs for $P$. These notions correspond to similar definitions in [2],[6], [9] and [7]. 
Dissimilar Sets of Variables Given an assertion $B$ over $P^{\prime}$, and another set $P \subseteq P^{\prime}$, the projection of $B$ onto $P$, written $\operatorname{proj}_{P^{\prime}, P}(B)$, is the set of runs obtained when each run in $B$ is restricted to the set of variables $P$. Using notion of relational algebra [20] we have $\operatorname{proj}_{P}(B)=\pi_{P}(B)$.

Given an assertion $B$ over $P^{\prime}$, and another set $P \supseteq P^{\prime}$, the inverse projection of $B$ onto $P$, written $\operatorname{proj}_{P}^{-1}(B)$, is the set of runs obtained when each run in $B$ is extended with all possible runs for $P \backslash P^{\prime}$. We can also express this as that the projection of all runs in $\operatorname{proj}_{P}^{-1}(B)$ onto $P^{\prime}$ must be in $B$, i.e. $\operatorname{proj}_{P}^{-1}(B)=$ $\left\{x_{P} \mid x_{P}\right.$ is a run for $\left.P, \operatorname{proj}_{P^{\prime}}\left(\left\{x_{P}\right\}\right) \subseteq B\right\}$.

Receptiveness of Assertions Let $\Omega_{P}$ be the set of all possible runs for $P$. An assertion $B$ is said to be $P$-receptive if $\operatorname{proj}_{P}(B)=\Omega_{P}$. This corresponds to $[2],[6]$, and [7] where the notion of receptiveness is described as the ability of an assertion to accept any history of values offered to a subset of its ports.

\subsection{Components and Contracts}

A contract $C$ modeled over a set of ordered variables $P$ is a pair of assertions $(A, G)$ where both $A$, the assumption, and $G$, the guarantee, are assertions over $P$. An implementation $M$, sometimes also called a design, modeled over a set of ordered variables $P^{\prime}$ is a pair $\left(P^{\prime}, B_{M}\right)$ where $B_{M} \neq \emptyset$ is an assertion over $P^{\prime}$.

A component $I$ is a tuple $\left(\mathcal{P}, \mathcal{M}_{\text {tot }}, \mathcal{C}_{t o t}, \mathcal{I}_{\text {sub }}\right)$, where

- $\mathcal{P}$ is a pair $\left(P_{u}, P_{c}\right)$ where $P_{u}$ and $P_{c}$ are non-empty mutually disjoint ordered sets of variables;

- $\mathcal{M}_{\text {tot }}$ is a set of $P_{u}$-receptive implementations $\left\{M_{1}, \ldots, M_{N_{I}}\right\}$ where each $M_{i}$ is modeled over $P_{u} \cup P_{c}$;

$-\mathcal{C}_{\text {tot }}$ is a set of contracts $\left\{C_{1}, \ldots, C_{N_{C}}\right\}$ where each $C_{j}$ is modeled over $P_{u} \cup P_{c}$; and

- $\mathcal{I}_{\text {sub }}$ is a set of components $\left\{I_{1}, \ldots, I_{N_{\text {sub }}}\right\}$ where each component $I_{k}=$ $\left(\mathcal{P}^{k}, \mathcal{M}_{\text {tot }}^{k}, \mathcal{C}_{\text {tot }}^{k}, \mathcal{I}_{\text {sub }}^{k}\right)$ is a sub-component of $I$ and where $P_{c}^{k} \in \mathcal{P}^{k}$ of each sub-component and $P_{u}$ are mutually disjoint.

Any variable in $P_{u} \cup P_{c}$ is called a port of $I$. In addition, a port in $P_{u}$ is called uncontrolled and a port in $P_{c}$ is called controlled. As mentioned in [2], uncontrolled/controlled ports correspond to the typical classification ${ }^{5}$ of input/output ports, respectively.

Properties of Contracts A contract $C=(A, G)$ of a component with ports $P_{u} \cup P_{c}$ is said to be port-compatible if $A$ is $P_{c}$-receptive and port-consistent if $G$ is $P_{u}$-receptive as described in $[2,6,7]$, but where we add the prefix "port-" to avoid ambiguity in terminology with respect to ISO 26262 (see Sec. 5.4).

\footnotetext{
${ }^{5}$ The ports are explicitly partitioned into uncontrolled and controlled ports in the component instead of relying on profiles to partition the ports of implementations, contracts, and components as in [2], [6] and [7], although the same principles apply.
} 
For an implementation $M=\left(P, B_{M}\right)$ and a contract $C=(A, G)$ modeled over the same ports, $M$ is said to satisfy $C$, written $M \models C$, if

$$
A \cap B_{M} \subseteq G .
$$

In accordance with [8], given a port-compatible and -consistent contract $C=$ $(A, G)$ of a component $I$ with sub-components $I_{1}, \ldots, I_{N_{s u b}}$ where there exists a port-compatible and -consistent contract $C_{k}=\left(A_{k}, G_{k}\right)$ for each $I_{k}, C$ is said to dominate $\left\{C_{1}, \ldots, C_{N}\right\}$, if

$$
A \cap\left(\bigcap_{j=1, j \neq k}^{N_{s u b}} G_{j}\right) \subseteq A_{k} \text { for } k=1, \ldots, N_{\text {sub }}
$$

$$
A \cap\left(\bigcap_{k=1}^{N_{s u b}} G_{k}\right) \subseteq G
$$

where the assertions are extended to a common set of variables, as described in Sec. 4.1, prior to applying set-theoretic operations (e.g. $\cap$ ) or comparing assertions with relations (e.g. $\subseteq$ ).

\section{$5 \quad$ Structuring Safety Requirements in ISO 26262 using Contract Theory}

In order to specify contracts for an item and its elements, we first need to model an item and its environment as components as presented in Sec. 4.2. That is, using the FLD-system as an example, we model the item and its environment as two tuples $\left(\mathcal{P}^{\text {item }}, \mathcal{M}_{\text {tot }}^{\text {item }}, \mathcal{C}_{\text {tot }}^{\text {item }}, \mathcal{I}_{\text {sub }}^{\text {item }}\right)$ and $\left(\mathcal{P}^{e n v}, \mathcal{M}_{\text {tot }}^{\text {env }}, \mathcal{C}_{\text {tot }}^{\text {env }}, \mathcal{I}_{\text {sub }}^{\text {env }}\right)$, respectively where COO, CAN and ICL are elements in $\mathcal{I}_{\text {sub }}^{\text {item }}$, and fuel tank and EMS are elements in $\mathcal{I}_{\text {sub }}^{e n v}$, as shown in Fig. 2. We only model variables as ports to the components if we need to refer to them in an assumption or guarantee. For example, the indicated fuel volume, shown by the fuel gauge, is modeled as the controlled port indicatedFuelVolume [\%] of the item.

We assume that the item only implements the functionality of the FLDsystem. In reality, the ECU-systems (COO and ICL) also implement other functionalities; e.g. $\mathrm{COO}$ also implements Cruise Control. We also assume that a more general contract is already in place concerning power delivery to the ECUsystems and we therefore model the status of the ignition (ignition [Bool]) as a port on all ECU-systems.

\subsection{Characterizing Safety Goals as a Contract}

In this section, we show how we can characterize Safety Goals (SGs), i.e. a top-level safety requirements, in ISO 26262 using contracts, by specifying safety goals for an item as a guarantee, given explicit requirements on its environment, expressed by an assumption. To illustrate these principles, we specify a contract $C_{\text {item }} \in \mathcal{C}_{\text {tot }}^{\text {item }}$ for the FLD-system in Table 1 where the guarantee $G_{i t e m}$ is a Safety Goal, and the assumption $A_{\text {item }}$ expresses requirements on the environment of the item. The Safety Goal $G_{i t e m}$ can be interpreted as: the FLD-system 


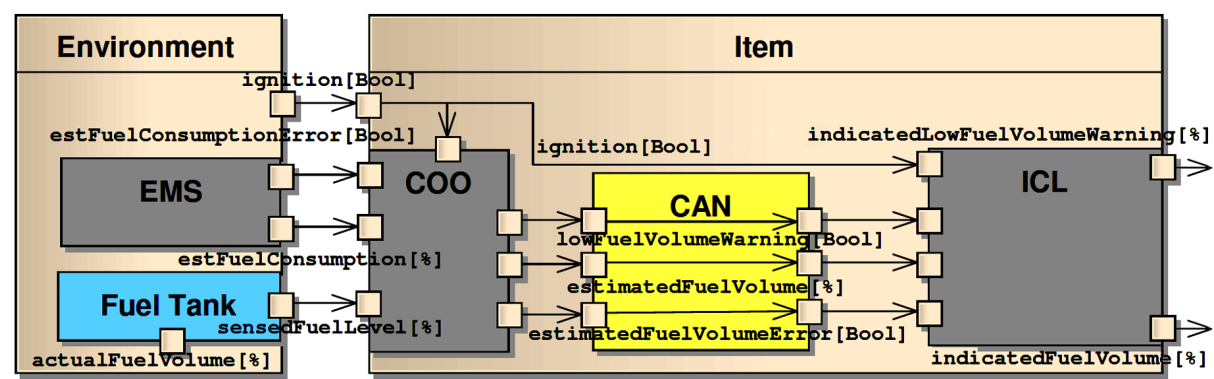

Fig. 2. A representation of the item (COO, CAN, and ICL) and the environment (Fuel Tank and EMS) modeled as components using a SysML internal block-diagram. Inputs and outputs to the blocks correspond to uncontrolled and controlled ports, respectively. Since we assumed that CAN delivers its signals immediately and with perfect accuracy (see Sec. 5.2), we choose to model signals over CAN as one variable.

shall not provide misguiding information to the driver while driving. The assumption $A_{\text {item }}$ can be interpreted as: the fuel sensor shall be correctly installed and that the EMS shall provide an accurate estimate of the fuel consumption. The contract $C_{i t e m}$ therefore imposes, through $A_{i t e m}$, its requirements on the environment in order to achieve its Safety Goal. That is, if e.g. the fuel sensor is installed incorrectly, the item cannot guarantee the Safety Goal $G_{i t e m}$.

In Table 1, we formalize the notion of driving as a state of the vehicle when the fuel volume derivative is less than zero. We further let the Safety Goal $G_{F L D S}^{i t e m}$ be characterized by three safe states (while driving): the indicatedFuelVolume [\%], shown by the fuel gauge, is less than actualFuelVolume [\%]; the warning lowFuelVolumeWarning [Bool] is functioning correctly; and the indicatedFuelVolume [\%] is less than $0 \%$.

In assumption $A_{\text {item }}$ in Table 1, we assume a relation between actualFuelVolume [\%] and sensedFuelLevel [\%] from the fuel sensor, and between the derivative of actualFuelVolume [\%] and the estimated fuel consumption (estFuelConsumption $[\%]$ ) and its signal status (estFuelConsumptionError $[\%]$ ), respectively. The assumed relation between ignition [Bool] and the fuel volume derivative may seem redundant; it is, however, motivated in Sec. 5.4.

\subsection{Characterizing Functional Safety Requirements as Contracts}

In the same manner in which we characterized a Safety Goal by a contract in Sec. 5.1, we can characterize Functional Safety Requirements (FSRs) as contracts for the elements of an item. Applying this concept to the FLD-system results in three contracts $C_{C O O}, C_{C A N}$, and $C_{I C L}$ with FSRs as guarantees, given assumptions on their environment, as shown in Tables 2,3 , and 4 , respectively.

The FSR $G_{C O O}$ in Table 2 expresses that COO shall: provide an estimate of the fuel volume (estimatedFuelVolume [\%]) that is less than or equal to actualFuelVolume [\%] and a Boolean signal (lowFuelVolumeWarning [Bool]) 
indicating if actualFuelVolume [\%] is below 10\%; or set the signal status of the estimated fuel volume (estimatedFuelVolumeError [Bool]) to erroneous (true). Note that assumption $A_{C O O}$ in Table 2 is identical to $A_{\text {item }}$ in Table 1.

As indicated in Table 3, the set of FSRs $G_{C A N}$ expresses that all CANsignals are delivered immediately and with perfect accuracy. This is of course not realistic, but for this system, safety aspects are not highly affected due to slight delays over CAN and such a simplification is therefore deemed to be justifiable. CAN does not impose any requirements on its environment and the assumption $A_{C A N}$ is thus receptive to its input ports (see Sec. 4.1).

As presented in Table 4, ICL basically acts like an actuator without any substantial additional logic. It assumes that the CAN-signals sent from $\mathrm{COO}$ are delivered immediately with perfect accuracy, as modeled by $A_{I C L}$. When ignition [Bool] is on, $G_{I C L}$ expresses that the fuel gauge shall display estimatedFuelVolume [\%] as indicatedFuelVolume [\%] and lowFuelVolumeWarning [Bool] as indicatedLowFuelVolumeWarning [Bool] in case the signal status of the estimated fuel volume is valid (estimatedFuelVolumeError [Bool] = false). In case it is erroneous, the fuel gauge shall indicate a value below $0 \%$.

\subsection{Modification of the Contract Theory of SPEEDS and its Implications}

In Table 1 and 2, it can be noted that the assumptions $A_{\text {item }}$ and $A_{C O O}$, and guarantees $G_{i t e m}$ and $G_{C O O}$ are not limited to the system boundary, i.e. to the ports, of the item and COO, respectively. This is necessary since ISO 26262 requires that properties of an environment, not limited to the system boundary of the item/element, are taken into consideration, see e.g. requirement 5.4.2e) from 3-5 Item definition in Sec. 3. Hence, the limitation that a contract must be modeled over the ports of its component (see Sec. 4.2) has been relaxed.

As a result of this, using the requirements on the low fuel volume warning in Table 1 as an example, we are able to express the (sub-)requirement in $G_{\text {item }}$ that the warning shall be active when the actual fuel volume is below 10\%, given the assumption $A_{\text {item }}$ that EMS shall provide an accurate estimation of the fuel consumption and that the fuel sensor has been installed correctly. If we, in contrast, restrict contracts to be modeled over the ports of its component, it is impossible to express the assumption $A_{\text {item }}$ and the Safety Goal $G_{i t e m}$, since the actual fuel volume is not a port of the item, see Fig. 2. In the case of $A_{\text {item }}$, for example, we cannot express that there is in fact a relation between the signal provided by the fuel sensor and the actual fuel volume.

This modification has a slight impact on the properties of contracts as presented in Sec. 4.2. The relation in (1) is generalized in the sense that the constraint that $B_{M}, A$, and $G$ must be modeled over the same ports is removed. An implementation $B_{M}$ and the assertions $A$ and $G$ must therefore be extended to a common set of variables before applying intersection and comparing with the subset relation (see Sec. 4.1). We let $P_{\Omega}$ be the universal set of all ports and $P_{\text {intP }}$ the set of all ports of all sub-components $I_{i}$ of $I$ and of all subcomponents of each $I_{i}$, and so forth. We say that a contract $C=(A, G)$ of a 
Table 1. Contract of the item, characterizing the Safety Goal for the FLD-system

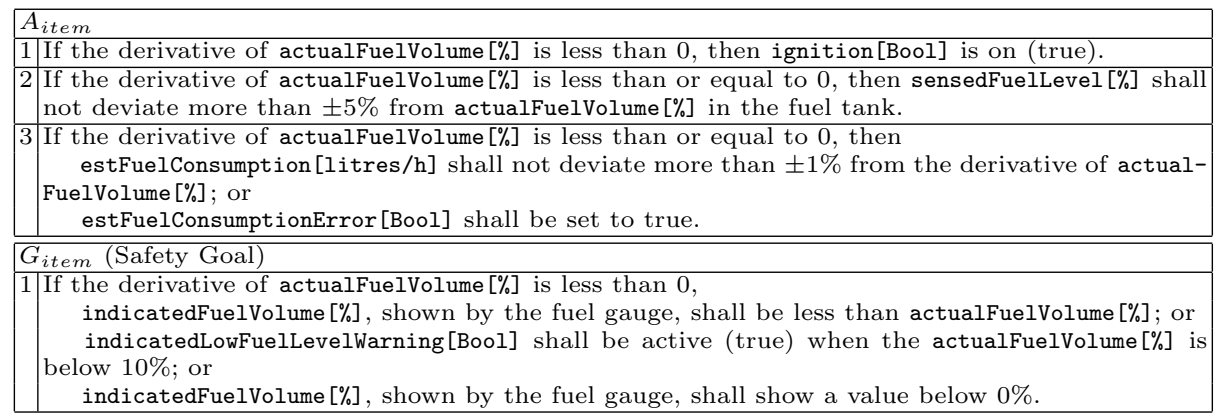

Table 2. Contract for COO, characterizing a Functional Safety Requirement for the FLD-system

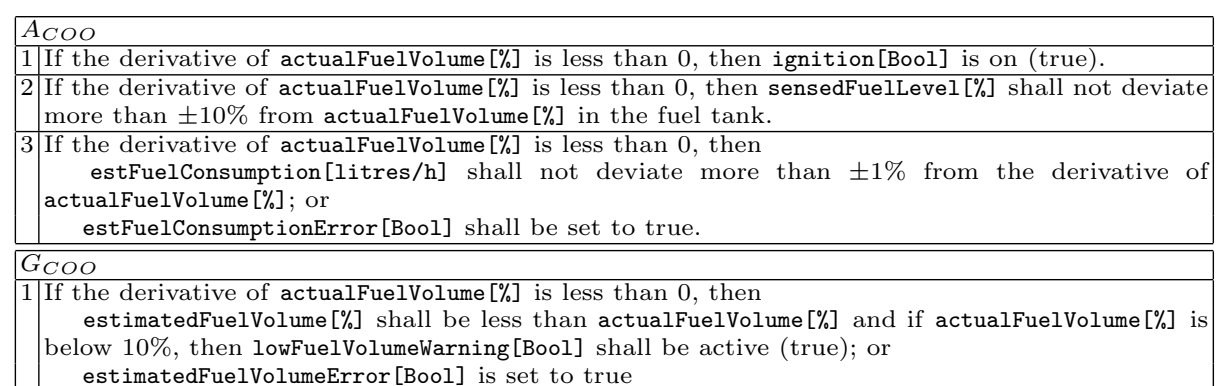

below $10 \%$, then lowFuelVolumeWarning [Bool] shall be active (true); or

estimatedFuelVolumeError [Bool] is set to true

Table 3. Contract for CAN, characterizing Functional Safety Requirements for the FLD-system

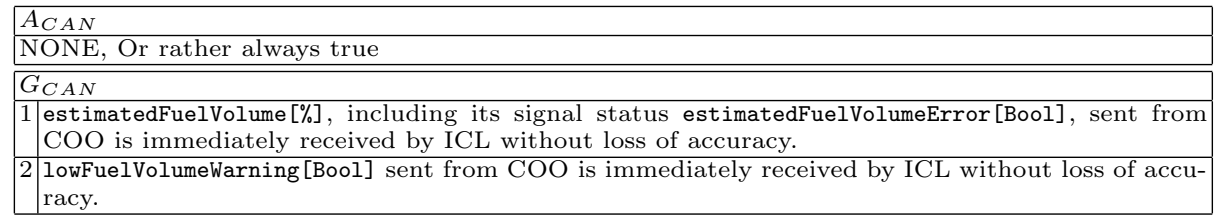

Table 4. Contract for ICL, characterizing Functional Safety Requirements for the FLD-system

\begin{tabular}{|c|c|}
\hline & $I C L$ \\
\hline & $\begin{array}{l}\text { estimatedFuelVolume }[\%] \text {, including its signal status estimatedFuelVolumeError [Bool], sent from } \\
\text { COO is immediately received by ICL without loss of accuracy. }\end{array}$ \\
\hline & $\begin{array}{l}\text { lowFuelVolumeWarning [Bool] sent from COO is immediately received by ICL without loss of accu- } \\
\text { racy. }\end{array}$ \\
\hline & $I C L$ \\
\hline & $\begin{array}{l}\text { If ignition [Bool] is on (true) and estimatedFuelVolumeError [Bool] is false, then } \\
\text { indicatedFuelVolume[\%], shown by the fuel gauge, shall correspond to the estimatedFuelVol- } \\
\text { ume [\%]; or } \\
\text { indicatedFuelVolumeWarning[Bool] shall correspond to lowFuelVolumeWarning[Bool]; }\end{array}$ \\
\hline & If ignition [Bool] is on and estimatedFuelVolumeError [Bool] is true, then indicatedFuelVolume [\%] \\
\hline
\end{tabular}


component $I$ is port-consistent if $G$ is $P_{\Omega} \backslash P_{c}$-receptive and port-compatible if $A$ is $P_{c} \cup\left(P_{\text {int } P} \backslash P_{u}\right)$-receptive.

\subsection{Verififying Consistency and Completeness of Safety Requirements}

ISO 26262 is based on the principle of relying on requirements as the main source of information to enforce correctness of design and implementation throughout the development process. This amounts to verifying properties of requirements and of sets of requirements as mentioned in 8-6 The specification and management of safety requirements in [1]. One of these properties is consistency of requirements, which means that "an individual requirement does not contradict itself" (internal consistency) and that "a set of requirements do not contradict each other"[1] (external consistency). Another property is completeness, which means that "the safety requirements at one requirement level fully implement all safety requirements of the previous level" [1].

We show, in the following, that the contract theory of SPEEDS supports the verification of consistency and completeness of safety requirements - as required by ISO 26262. As indicated in Sec. 5.1 and 5.2, we consider a safety requirement or a set of safety requirements as a guarantee $G$ of a contract $C$ for an element/item, where $A$ expresses the requirements on its environment. With inspiration from [9], we consider a safety requirement $G$ of a contract $C$, to be internally consistent if $G \neq \emptyset$, i.e. if there exist at least one run in $G$. As indicated in Theorem 1, internal consistency of a safety requirement $G$ can be ensured through a port-consistent contract $(A, G)$ (see [21] for proof).

Theorem 1. If a contract $C=(A, G)$ of a component I is port-consistent (see Sec. 4.2 and 5.3), then the safety requirement $G$, is internally consistent.

The dominance property of contracts in Sec. 4.2 can be used to support the verification of completeness and external consistency as indicated in Theorem 2 (see [21] for proof). Since we consider the use of safety requirements in a context of contracts, external consistency does not only amount to showing that a set of safety requirements $\left\{G_{1}, \ldots, G_{N}\right\}$ are not contradictory, but also not contradictory with respect to their corresponding assumptions $\left\{A_{1}, \ldots, A_{N}\right\}$. We thus consider a set of safety requirements $\left\{G_{1}, \ldots, G_{N}\right\}$ with corresponding assumptions $\left\{A_{1}, \ldots, A_{N}\right\}$ where $A_{i}, G_{i} \in$ a contract $C_{i}$ to be external consistent if $\left(\bigcap_{i=1}^{N_{\text {sub }}} A_{i}\right) \cap \bigcap_{i=1}^{N_{\text {sub }}} G_{i} \neq \emptyset$.

We consider completeness for a scenario where we have a contract $C=(A, G)$ of a component $I$ with sub-components $I_{1}, \ldots, I_{N_{\text {sub }}}$ and there exists a contract $C_{k}=\left(A_{k}, G_{k}\right)$ for each $I_{k}$. We say that a set of safety requirements $\left\{G_{1}, \ldots, G_{N}\right\}$ is complete with respect to $G$ if for any implementation $M_{k}$ of each $I_{k}$ that satisfies its contract, i.e. if $M_{k}=C_{k}$, then $A \cap\left(\bigcap_{k=1}^{N_{s u b}} M_{k}\right) \subseteq G$.

Theorem 2. Let $C=(A, G)$, be a port-compatible and -consistent contract of a component I with sub-components $I_{1}, \ldots, I_{N_{s u b}}$, and $C_{k}=\left(A_{k}, G_{k}\right)$ be 
a port-compatible and -consistent contract of each $I_{k}$. Furthermore, if $G$ and $\left\{G_{1}, \ldots, G_{N_{\text {sub }}}\right\}$ are safety requirements, then $\left\{G_{1}, \ldots, G_{N_{s u b}}\right\}$ are externally consistent and complete with respect to $G$ if $C$ dominates $\left\{C_{1}, \ldots, C_{N}\right\}$.

If we investigate the FLD-system example, we see that all contracts in Tables $1,2,3$, and 4 are port-consistent and -compatible since they respect the constraints in Sec. 4.2 and 5.3, see [21] for further clarification. Internal consistency is hence ensured through Theorem 1 . We can verify the external consistency and completeness of the set of FSRs $G_{C O O}, G_{C A N}$, and $G_{I C L}$ in Tables 2, 3, and 4 with respect to the Safety Goal $G_{\text {Item }}$ in Table 1 through Theorem 2, if we can show that:

$$
\begin{aligned}
& A_{\text {Item }} \cap\left(\bigcap_{j \neq i} G_{j}\right) \subseteq A_{i} \text { for } i=C O O, C A N, I C L \text { and } \\
& A_{\text {Item }} \cap\left(G_{C O O} \cap G_{C A N} \cap G_{I C L}\right) \subseteq G_{\text {Item }} .
\end{aligned}
$$

Relation (4) is trivially true, since $A_{\text {Item }}=A_{C O O}, G_{C A N}=A_{I C L}$, and $A_{C A N}$ is receptive to the set of universal ports $P_{\Omega}$. Concerning relation (5), since we have assumed that the fuel volume derivative is always negative when ignition [Bool] is on (see $A_{\text {Item }}$ in Table 1), we can conclude that when indicatedFuelVolume [\%] is less than zero and ignition [Bool] is on (see $G_{I C L}$ in Table 4) corresponds to a safe state of the item, see $G_{\text {Item }}$ in Table 1. If estimatedFuelVolumeError $[\%]=$ false and ignition [Bool] is on, ICL will either display the fuel warning (indicatedLowFuelVolumeWarning [Bool]) in case actualFuelVolume $[\%]<10 \%$ or indicatedFuelVolume $[\%]$ that is less than actualFuelVolume [\%], since this is guaranteed by the contracts of CAN and COO (see Tables 2 and 3). These states both correspond to safe states of the item and hence relation (5) is also true. Through Theorem 2, we can hence claim that the FSRs, expressed by $G_{C O O}, G_{C A N}$, and $G_{I C L}$, are externally consistent and complete with respect to the Safety Goal, expressed by $G_{i t e m}$.

\section{Conclusions}

We have shown in Sec. 5.1 and 5.2 that safety requirements can be characterized by contracts for an item and its elements, with guarantees that constitute the safety requirements, given explicit requirements on their environments as assumptions. A Contract therefore enriches a safety specification for an item/element by explicitly declaring what each element/item expects from the environment to ensure that the safety requirements are satisfied.

We have also shown in Sec. 5.4 that consistency and completeness of safety requirements can be ensured through verifying the dominance property of contracts.

However, these achievements were only made possible due to a modification of the contract theory of SPEEDS, as presented in Sec. 5.3. The modification 
relaxes the constraint that a contract must be modeled over the ports of its component.

We hence conclude that the principles of contracts provide a suitable foundation to structure safety requirements in ISO 26262.

\section{Annex}

In this paper and in the proofs below, we assume that all runs are sorted according to a global ordering with respect to the variables. We use an operator $\cup_{*}$ between runs for disjoint sets of variables as a modification of the union operator of unordered sets. That is, if $b_{1}, \ldots, b_{N}$ are $N$ runs for $N$ disjoint sets of variables, respectively, then $b_{1} \cup_{*} \ldots \cup_{*} b_{N}$ is a run where all elements for each run $b_{k}$ for $k=1, \ldots, N$ are also in $b_{1} \cup_{*} \ldots \cup_{*} b_{N}$ and where $b_{1} \cup_{*} \ldots \cup_{*} b_{N}$ is, as mentioned, sorted according to a global ordering with respect to the variables. We also use an operator $\subseteq_{*}$ between runs as a modification of the subset relation on unordered sets, i.e. if $b=\left(\ldots, x_{k}, \ldots\right)$ and $b^{\prime}=\left(\ldots, y_{k}, \ldots\right)$ are runs, then $b \subseteq b^{\prime}$ if $\forall k, x_{k} \in b . \exists y_{k} \in b^{\prime} . x_{k}=y_{k}$.

Theorem 1. If a contract $C=(A, G)$ of a component $I$ is port-consistent (see Sec. 4.2 and 5.3) and $G$ is a safety requirement, then $G$ is internally consistent.

Proof.

$C=(A, G)$ of $I$ with ports $P_{u} \cup P_{c}$ is port-consistent [Premise]

$G$ is $P_{\Omega} \backslash P_{c}$-receptive [Def. Port-consistency (see Section 5.3) and (1)]

$\operatorname{proj}_{P_{\Omega} \backslash P_{c}}(G)=\Omega_{P_{\Omega} \backslash P_{c}}$ [Def. Receptiveness (see Section 4.1) and (2)]

$\forall b, b$ is a run for $P_{\Omega} \backslash P_{c} \cdot\left(b \in \operatorname{proj}_{P_{\Omega} \backslash P_{c}}(G)\right)[(3)]$

$\exists b^{\prime}, b^{\prime}$ is a run for $P_{c} .\left(b^{\prime} \in \operatorname{proj}_{P_{c}}(G)\right)[(3)]$

$\forall b, b$ is a run for $P_{\Omega} \backslash P_{c} \cdot \exists b^{\prime}, b^{\prime}$ is a run for $P_{c} .\left(b \cup_{*} b^{\prime}\right) \in G[(4)$ and (5)]

$$
G \neq \emptyset[(6)]
$$

Theorem 2. Let $C=(A, G)$, be a port-compatible and-consistent contract of a component $I$ with sub-components $I_{1}, \ldots, I_{N_{s u b}}$, and $C_{k}=\left(A_{k}, G_{k}\right)$ be a portcompatible and -consistent contract of each $I_{k}$ for $k=1, \ldots, N_{\text {sub }}$. Furthermore, if $G$ and $\left\{G_{1}, \ldots, G_{N_{\text {sub }}}\right\}$ are safety requirements, then $\left\{G_{1}, \ldots, G_{N_{\text {sub }}}\right\}$ are externally consistent and complete with respect to $G$ if $C$ dominates $\left\{C_{1}, \ldots, C_{N}\right\}$.

Proof.

$$
C \text { dominates }\left\{C_{1}, \ldots, C_{N_{s u b}}\right\} \text { [Premise] }
$$

$C=(A, G)$ of $I$ with ports $P_{u} \cup P_{c}$ is port-consistent and -compatible and $\mathrm{G}$ is a safety requirement [Premise] $C_{k}=\left(A_{k}, G_{k}\right)$ of $I_{k}$ with ports $P_{u}^{k} \cup P_{c}^{k}$ is port-consistent and port-compatible and $G_{k}$ is a safety requirement for $k=1, \ldots, N_{\text {sub }}$ 
[Def. Dominance (see Section 4.2) and (1)]

$$
A \cap\left(\bigcap_{k=1}^{N_{\text {sub }}} G_{k}\right) \subseteq A_{k} \text { for } k=1, \ldots, N_{\text {sub }}
$$

[Def. Intersection and Subset and (5)]

$$
\begin{array}{r}
A \cap\left(\bigcap_{k=1}^{N_{\text {sub }}} G_{k}\right) \subseteq \bigcap_{k=1}^{N_{\text {sub }}} A_{k} \text { [Def. Intersection and Subset and (6)] } \\
A \cap\left(\bigcap_{k=1}^{N_{s u b}} G_{k}\right) \neq \emptyset \Rightarrow \bigcap_{k=1}^{N_{s u b}} A_{k} \cap\left(\bigcap_{k=1}^{N_{s u b}} G_{k}\right) \neq \emptyset[(7)]
\end{array}
$$

$G_{k}$ is $P_{\Omega} \backslash P_{c}^{k}$-receptive for $k=1, \ldots, N_{\text {sub }}$

[Def. Port-consistency (see Section 5.3) and (3)]

$$
\operatorname{proj}_{P_{\Omega} \backslash P_{c}^{k}}\left(G_{k}\right)=\Omega_{P_{\Omega} \backslash P_{c}^{k}} \text { for } k=1, \ldots, N_{\text {sub }}
$$

[Def. Receptiveness (see Section 4.1) and (9)]

$\forall b, b$ is a run for $P_{\Omega} \backslash P_{c}^{k} . \exists b^{\prime}, b^{\prime}$ is a run for $P_{c}^{k} .\left(b \cup_{*} b^{\prime}\right) \in G_{k}$

$$
\text { for } k=1, \ldots, N_{\text {sub }}[(10)]
$$

$\forall b^{\prime}, b^{\prime}$ is a run for $P_{c}^{k} \cdot \exists b^{\prime \prime}, b^{\prime \prime}$ is a run for $P_{\Omega} \backslash P_{c}^{j \neq k}$.

$$
b^{\prime} \subseteq_{*} b^{\prime \prime} \text { for } k, j=1, \ldots, N_{\text {sub }}
$$

[Def. Component (see Section 4.2) and (4)]

$\forall b, b$ is a run for $P_{\Omega} \backslash \bigcup_{k=1}^{N_{s u b}} P_{c}^{k} . \exists b_{1}^{\prime}, \ldots, b_{N_{s u b}}^{\prime}, b_{k}^{\prime}$ is a run for $P_{c}^{k}$.

$\left(b \cup_{*} b_{1}^{\prime} \cup_{*} \ldots \cup_{*} b_{N_{s u b}}^{\prime}\right) \in \bigcap_{k=1}^{N_{s u b}} G_{k}$ [Def. Intersection, (12), and (11) ]

$$
A \text { is } P_{c} \cup\left(P_{\text {int } P} \backslash P_{u}\right) \text {-receptive }
$$

[Def. Port-consistency (see Section 5.3) and (3)]

$$
\operatorname{proj}_{P_{c} \cup\left(P_{\text {int } P} \backslash P_{u}\right)}(A)=\Omega_{P_{c} \cup\left(P_{\text {int } P} \backslash P_{u}\right)}
$$

[Def. Receptiveness (see Section 4.1) and (14)] (15) $\forall b_{\text {int }}, b_{\text {int }}$ is a run for $P_{c} \cup\left(P_{\text {int } P} \backslash P_{u}\right)$.

$\exists b_{\text {ext }}, b_{\text {ext }}$ is a run for $P_{\Omega} \backslash\left(P_{c} \cup\left(P_{\text {int } P} \backslash P_{u}\right)\right)$.

$$
\left(b_{\text {int }} \cup_{*} b_{\text {ext }}\right) \in A[(15)] \quad(16)
$$

$\forall b_{\text {ext }}, b_{\text {ext }}$ is a run for $P_{\Omega} \backslash\left(P_{c} \cup\left(P_{\text {int } P} \backslash P_{u}\right)\right)$. 
$\exists b, b$ is a run for $P_{\Omega} \backslash \bigcup_{k=1}^{N_{s u b}} P_{c}^{k} \cdot b_{e x t} \subseteq_{*} b$ [Def. Component and (4)] $\forall b_{1}^{\prime}, \ldots, b_{N_{s u b}}^{\prime}, b_{k}^{\prime}$ is a run for $P_{c}^{k} . \exists b_{i n t}, b_{i n t}$ is a run for $P_{c} \cup\left(P_{\text {intP }} \backslash P_{u}\right)$.

$\left(b_{1}^{\prime} \cup_{*} \ldots \cup_{*} b_{N_{s u b}}^{\prime}\right) \subseteq_{*} b_{\text {int }}$ [Def. Component and (4)]

$\exists b, b_{1}^{\prime}, \ldots, b_{N_{s u b}}^{\prime}, b$ is a run for $P_{\Omega} \backslash \bigcup_{k=1}^{N_{s u b}} P_{c}^{k}$ and $b_{k}^{\prime}$ is a run for $P_{c}^{k}$.

$$
\left(b \cup_{*} b_{1}^{\prime} \cup_{*} \ldots \cup_{*} b_{N_{s u b}}^{\prime}\right) \in A \cap\left(\bigcap_{k=1}^{N_{s u b}} G_{k}\right)
$$

[Def. Intersection, (13), (16), (17), and (18)] (19)

$$
\begin{aligned}
& A \cap\left(\bigcap_{k=1}^{N_{s u b}} G_{k}\right) \neq \emptyset[19] \\
& \bigcap_{k=1}^{N_{\text {sub }}} A_{k} \cap\left(\bigcap_{k=1}^{N_{\text {sub }}} G_{k}\right) \neq \emptyset \text { [Def. Implication elimination, (8), and (20)] } \\
& M_{1}^{0}, \ldots, M_{N_{s u b}}^{0} \text { where } M_{k}^{0} \text { is an implementation of } I_{k}(22) \\
& M_{k}^{0} \models C_{k} \text { for } k=1, \ldots, N_{\text {sub }} \text { [Assumption] (23) } \\
& A \cap\left(\bigcap_{k=1}^{N_{s u b}} G_{k}\right) \subseteq G \text { [Def. Dominance on (1)] } \\
& A \cap\left(\bigcap_{k=1}^{N_{s u b}} A_{k}\right) \cap\left(\bigcap_{i=1}^{N_{s u b}} B_{M_{k}^{0}}\right) \subseteq G \\
& A \cap\left(\bigcap_{k=1}^{N_{s u b}} B_{M_{k}^{0}}\right) \subseteq G[(5), \text { Def. Subset, and (25)] } \\
& \forall M_{1}, \ldots, M_{N_{s u b}}, M_{k} \text { of } I_{k} .\left(M_{k} \models C_{k} \text { for } k=1, \ldots, N_{s u b}\right) \Rightarrow \\
& A \cap\left(\bigcap_{k=1}^{N_{s u b}} B_{M_{k}}\right) \subseteq G
\end{aligned}
$$

[Existential and Implication intr. (23)-(26) and (24)-(26)] (27)

\section{References}

1. ISO: 26262 - "Road vehicles-Functional safety" (2011)

2. Benveniste, A. et al.: Multiple Viewpoint Contract-Based Specification and Design. In Boer, F. S. et al., eds.: Formal Methods for Components and Object. SpringerVerlag, Berlin, Heidelberg (2008) 200-225

3. Blanquart, J.-P. et al.: Towards Cross-Domains Model-Based Safety Process, Methods and Tools for Critical Embedded Systems: The CESAR Approach. In: Com- 
puter Safety, Reliability, and Security. Volume 6894 of Lecture Notes in Computer Science. Springer Berlin Heidelberg (2011) 57-70

4. Baumgart, A. et al.: A Model-Based Design Methodology with Contracts to Enhance the Development Process of Safety-Critical Systems. In: Software Technologies for Embedded and Ubiquitous Systems. Volume 6399 of Lecture Notes in Computer Science. Springer Berlin Heidelberg (2011) 59-70

5. Damm, W., B. Josko, and T. Peinkamp: Contract Based ISO CD 26262 Safety Analysis. In: Safety-Critical Systems, 2009. SAE (2009)

6. Sangiovanni-Vincentelli, A. L., W. Damm, and R. Passerone: Taming Dr. Frankenstein: Contract-Based Design for Cyber-Physical Systems. Eur. J. Control 18(3) (2012) 217-238

7. Benveniste, A., B. Caillaud, and R. Passerone: Multi-Viewpoint State Machines for Rich Component Models (2008)

8. Graf, S., and S. Quinton: Contracts for BIP: Hierarchical Interaction Models for Compositional verification (2007)

9. Benveniste, A. et al.: Contracts for the Design of Embedded Systems. Part II: Theory. http://www.irisa.fr/distribcom/benveniste/pub/ (march 2013)

10. Meyer, B.: Applying "Design by Contract". IEEE Computer 25 (1992) 40-51

11. Hoare, C. A. R.: An Axiomatic Basis for Computer Programming. Commun. ACM 12(10) (October 1969) 576-580

12. Dijkstra, E. W.: Guarded Commands, Nondeterminacy and Formal Derivation of Programs. Commun. ACM 18(8) (August 1975) 453-457

13. Giese, H.: Contract-based Component System Design. In: Thirty-Third Annual Hawaii Int. Conf. on System Sciences (HICSS-33), Maui, IEEE Press (2000)

14. Sun, X. et al.: Contract-based System-Level Composition of Analog Circuits. In: Design Automation Conf., 2009. DAC '09. 46th ACM/IEEE. (july 2009) 605-610

15. Damm, W.: Controlling Speculative Design Processes Using Rich Component Models. In: Application of Concurrency to System Design, 2005. ACSD 2005. Fifth International Conference on. (june 2005) 118 - 119

16. Back, R.-J., and J. V. Wright: Contracts, Games and Refinement. In: Information and Computation, Elsevier (1997) 200-0

17. Alfaro, L. D., and T. A. Henzinger: Interface Theories for Component-based Design, Springer-Verlag (2001) 148-165

18. Dill, D. L.: Trace Theory for Automatic Hierarchical Verification of SpeedIndependent Circuits. In: Proceedings of the fifth MIT conference on Advanced research in VLSI, Cambridge, MA, USA, MIT Press (1988) 51-65

19. Negulescu, R.: Process Spaces. In: Proceedings of the 11th Int. Conf. on Concurrency Theory. CONCUR '00, London, UK, UK, Springer-Verlag (2000) 199-213

20. Codd, E. F.: A Relational Model of Data for Large Shared Data Banks. Commun. ACM 13(6) (June 1970) 377-387

21. Westman, J., M. Nyberg, and M. Törngren: Structuring Safety Requirements in ISO 26262 using Contract Theory. Technical Report TRITA MMK 2013:04, KTH (March 2012) 